\title{
Realized Stochastic Volatility Models with \\ Generalized Gegenbauer Long Memory
}

Complutense de Análisis Económico

\section{Manabu Asai}

Faculty of Economics Soka University, Japan

\section{Michael McAleer}

Department of Quantitative Finance National Tsing Hua University, Taiwan and Discipline of Business Analytics University of Sydney Business School, Australia

And Econometric Institute Erasmus School of Economics

Erasmus University Rotterdam, The Netherlands and

Department of Quantitative Economics Complutense University of Madrid, Spain

And Institute of Advanced Sciences Yokohama National University, Japan

\section{Shelton Peiris}

School of Mathematics and Statistics

University of Sydney, Australia

\begin{abstract}
In recent years fractionally differenced processes have received a great deal of attention due to their exibility in nancial applications with long memory. In this paper, we develop a new realized stochastic volatility (RSV) model with general Gegenbauer long memory (GGLM), which encompasses a new RSV model with seasonal long memory (SLM). The RSV model uses the information from returns and realized volatility measures simultaneously. The long memory structure of both models can describe unbounded peaks apart from the origin in the power spectrum. Forestimating the RSV-GGLM model, we suggest estimating the location parameters for the peaks of the power spectrum in the rst step, and the remaining parameters based on the Whittle likelihood in the second step. We conduct Monte Carlo experiments for investigating the nite sample properties of the estimators, with a quasi-likelihood ratio test of RSV-SLM model against theRSV-GGLM model. We apply the RSV-GGLM and RSV-SLM model to three stock market indices. The estimation and forecasting results indicate the adequacy of considering general long memory.
\end{abstract}

Keywords Stochastic Volatility; Realized Volatility Measure; Long Memory; Gegenbauer Polynomial; Seasonality; Whittle Likelihood.

\section{JEL Classification C18, C21, C58.}

\section{Working Paper no 1726 \\ November, 2017}

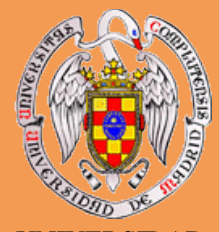

UNIVERSIDAD

COMPLUTENSE MADRID

ISSN: 2341-2356

WEB DE LA COLECCIÓN: http://www.ucm.es/fundamentos-analisis-economico2/documentos-de-trabajo-del-icaeWorking papers are in draft form and are distributed for discussion. It may not be reproduced without permission of the author/s. 


\title{
Realized Stochastic Volatility Models with Generalized Gegenbauer Long Memory*
}

\author{
Manabu Asai \\ Faculty of Economics \\ Soka University, Japan \\ Michael McAleer \\ Department of Quantitative Finance \\ National Tsing Hua University, Taiwan \\ and \\ Discipline of Business Analytics \\ University of Sydney Business School, Australia \\ and \\ Econometric Institute \\ Erasmus School of Economics \\ Erasmus University Rotterdam, The Netherlands \\ and \\ Department of Quantitative Economics \\ Complutense University of Madrid, Spain \\ and \\ Institute of Advanced Sciences \\ Yokohama National University, Japan \\ Shelton Peiris \\ School of Mathematics and Statistics \\ University of Sydney, Australia
}

November 2017

\footnotetext{
*The authors are most grateful to Yoshi Baba for very helpful comments and suggestions. The first author acknowledges the financial support of the Japan Ministry of Education, Culture, Sports, Science and Technology, Japan Society for the Promotion of Science, and the Australian Academy of Science. The second author is most grateful for the financial support of the Australian Research Council, National Science Council, Ministry of Science and Technology (MOST), Taiwan, and the Japan Society for the Promotion of Science. The third author acknowledges the support from the Faculty of Economics at Soka University.
} 


\begin{abstract}
In recent years fractionally differenced processes have received a great deal of attention due to their flexibility in financial applications with long memory. In this paper, we develop a new realized stochastic volatility (RSV) model with general Gegenbauer long memory (GGLM), which encompasses a new RSV model with seasonal long memory (SLM). The RSV model uses the information from returns and realized volatility measures simultaneously. The long memory structure of both models can describe unbounded peaks apart from the origin in the power spectrum. For estimating the RSV-GGLM model, we suggest estimating the location parameters for the peaks of the power spectrum in the first step, and the remaining parameters based on the Whittle likelihood in the second step. We conduct Monte Carlo experiments for investigating the finite sample properties of the estimators, with a quasi-likelihood ratio test of RSV-SLM model against theRSV-GGLM model. We apply the RSV-GGLM and RSV-SLM model to three stock market indices. The estimation and forecasting results indicate the adequacy of considering general long memory.
\end{abstract}

Keywords: Stochastic Volatility; Realized Volatility Measure; Long Memory; Gegenbauer Polynomial; Seasonality; Whittle Likelihood.

JEL Classification: C18, C21, C58. 


\section{Introduction}

For purposes of modeling financial time series, a stylized fact is that volatility has long memory. One of the popular approaches is to apply an autoregressive fractionally-integrated moving-average (ARFIMA) process to (log-)volatility. In the class of generalized autoregressive conditional heteroskedasticity (GARCH) models, Baillie, Bollerslev and Mikkelsen (1996) and Bollerslev and Mikkelsen (1996) developed the fractionally integrated GARCH (FIGARCH) and fractionally integrated Exponential GARCH (FIEGARCH) models, respectively. For stochastic volatility (SV) models, Breidt, Crato, and de Lima (1998) developed the long memory stochastic volatility (LMSV) model for unobserved log-volatility using asset return series, while Andersen et al. (2001, 2003), Pong et al. (2004), Koopman, Jungbacker, and Hol (2005), and Asai, McAleer, and Medeiros (2012) estimated LMSV models using daily realized volatility (RV). As an alternative to the ARFIMA model, Corsi (2009) suggested a heterogeneous autoregressive (HAR) model to approximate long memory using RV.

As extensions of the long memory structure in an ARFIMA process, seasonal (periodical) long memory, Gegenbauer processes, and their general class are considered. The Gegenbauer process is based on Gegenbauer polynomials, developed by Gray, Zhang, and Woodward (1989). While the spectral density of the ARFIMA process is unbounded at the origin, the Gegenbauer process has a peak at a different frequency, which is referred to as the Gegenbauer frequency. As suggested in Woofward, Cheng, and Gray (1998), general (or multifactor) Gegenbauer process has multiple (unbounded) peaks. The general Gegenbauer process encompasses seasonal long memory as a special case. While Bordignon, Caporin, and Lisi (2009) extended the FIGARCH and FIEGARCH models by accommodating seasonal long memory, Bordignon, Caporin, and Lisi (2007) developed the general Gegenbauer GARCH model. Although their focus is on investigating the long memory structure within a day, it may also be worth examining the general Gegenbauer process using daily realized volatility measure.

Fo modeling asset returns and realized volatility measure simultaneously, Hansen, Huang, and Shek (2012) suggested a realized GARCH framework (see also Hansen and Huang (2016)). The corresponding structure for SV is often referred to as the 'realized SV' (RSV) model, which is considered by Takahashi, Omori, and Watanabe (2009), Koopman and Scharth (2013), Shirota, 
Hizu, and Omori (2014), and Asai, Chang, and McAleer (2017), among others. Shirota, Hizu, and Omori (2014) and Asai, Chang, and McAleer (2017) accommodated the ARFIMA process in the volatility process. While Shirota, Hizu, and Omori (2014) use the Markov chain Monte Carlo technique, Asai, Chang, and McAleer (2017) estimated their model using the Whittle likelihood.

In this paper, we develop an RSV model with general Gegenbauer long memory. If the Gegenbauer frequencies of log-volatility are predetermined, we can use the Whittle likelihood estimator of Hosoya (1997) and Zaffaroni (2009) to estimate the RSV model, as in Asai, Chang, and McAleer (2017). However, the Gegenbauer frequencies are unknown, and so we use the non-parametric estimator of Hidalgo and Soulier (2004), as in Artiach and Arteche (2012), who investigated the long memory property of the level and variance of the number of sunspots.

The organization of the paper is as follows. Section 2 develops the RSV model with general Gegenbauer long memory, and discusses the differences from the model with seasonal long memory. Section 3 explains the estimation method based on the Whittle likelihood under predetermined Gegenbauer frequencies, and shows the approach of Hidalgo and Soulier (2004) for estimating and selecting the Gegenbauer frequencies. Section 3 provides the finite sample properties of these estimators, and the likelihood ratio statistic for testing the seasonal long memory against the general long memory. Section 4 presents empirical results using the daily returns and realized volatility measures of three stock indices, namely Standard \& Poors 500, FTSE 100, and Nikkei 225. Section 5 provides some concluding remarks.

\section{Realized SV with Generalized Gegenbauer Long Memory}

Let $y_{t}$ and $x_{t}$ denote the return and the $\log$ of realized volatility measure of a financial asset, repectively. We present the new realized SV model with generalized Gegenbauer long memory (RSV-GGLM), as follows:

$$
\begin{aligned}
& y_{t}=\varepsilon_{t} \exp \left(h_{t} / 2\right), \quad \varepsilon_{t} \sim N(0,1), \quad t=1, \ldots, T, \\
& x_{t}=h_{t}+v_{t}, \quad v_{t} \sim N\left(0, \sigma_{v}^{2}\right), \\
& \phi(L) P(L)\left(h_{t+1}-\mu\right)=\theta(L) \eta_{t}, \quad \eta_{t} \sim N\left(0, \sigma_{\eta}^{2}\right),
\end{aligned}
$$


where

$$
P(L)=\prod_{l=1}^{k}\left(1-2 \cos \left(\omega_{l}\right) L+L^{2}\right)^{d_{l}}(1-L)^{d},
$$

$\varepsilon_{t}, v_{t}$, and $\eta_{t}$ are independent processes, $L$ is the lag operator, $\phi(L)=1-\phi_{1} L-\ldots-\phi_{p} L^{p}$, and $\theta(L)=1+\theta_{1} L+\ldots+\theta_{q} L^{q}$. The log-volatility process $h_{t}$ is latent, while $y_{t}$ is observed. We assume the roots of $\phi(z)$ and $\theta(z)$ lie outside the unit circle to ensure stationarity and invertibility of $\left\{h_{t}\right\}$, respectively. Equation (3) is known as the $k$-factor Gegenbauer process or generalized exponential model. For the stationarity of long memory, we assume $|d|<1 / 2,\left|d_{l}\right|<1 / 2$, and $0<\omega_{l}<\pi$ (see Woodward, Cheng, and Gray (1998), and McElroy and Holan (2012)). We exclude $(1+L)^{d_{k+1}}$, as it is rare to find such a case in the analysis of financial time series.

By excluding the data of $x_{t}$, the model reduces to the class of generalized long memory SV models, encompassing the long memory SV model of Breidt, Crato, and de Lima (1998) with $k=0$, and the Gegenbauer ARMA SV (GARMASV) model of Artiach and Arteche (2012) with $k=1$ and $d=0$. Furthermore, the RSV-GGLM model extends the long memory part of the realized SV models of Shirota, Hizu, and Omori (2014) and Asai, Chang, and McAleer (2017). Note that we do not consider asymmetric effects and heavy-tails, unlike Shirota, Hizu, and Omori (2014) and Asai, Chang, and McAleer (2017), in order to concentrate on the specification and estimation of various long memory structures.

To consider the structure of the RSV-GGLM model, we start from a simple Gegenbauer process. When $k=1$ and $d=0$, we can write equation (3) as:

$$
h_{t+1}=\mu+\left(1-2 \cos \left(\omega_{1}\right) L+L^{2}\right)^{-d_{1}}[\phi(L)]^{-1} \theta(L) \eta_{t},
$$

which is known as the Gegenbaour process, named after the Gegenbauer polynomials defined by $\left(1-2 \cos \left(\omega_{1}\right) z+z^{2}\right)^{-d_{1}}=\sum_{j=0}^{\infty} b_{j} z^{j}$. The power spectrum of the Gegenbauer process is given by:

$$
f_{h}(\lambda)=\frac{\sigma_{\eta}^{2}}{2 \pi}\left[2\left(\cos \lambda-\cos \omega_{1}\right)\right]^{-2 d_{1}} g_{h}(\lambda),-\pi<\lambda<\pi,
$$

where $g_{h}(\lambda)=\frac{\left|\theta\left(e^{-i \lambda}\right)\right|^{2}}{\left|\phi\left(e^{-i \lambda}\right)\right|^{2}}$ corresponds to the autoregressive and moving-average (ARMA) part. The power spectrum shows the long memory feature characterized by an unbounded spectrum at the Gegenbauer frequency $\omega_{1}$. By the structure, the RSV-GGLM model accommodates conventional long memory and multi-factor Gegenbauer long memory. 
At this stage, we should consider the difference between $P(L)$ and the seasonal long memory filter, $\left(1-L^{s}\right)^{d}$ (see Porter-Hudak (1990)). As discussed in Bordignon, Caporin, and Lisi (2008), we can decompose the seasonal filter, as in $P(L)$. For instance, if we consider a weekly pattern for daily data $(s=5)$, we obtain:

$$
\left(1-L^{5}\right)^{d}=(1-L)^{d}\left(1-2 \cos \left(\frac{2 \pi}{5}\right) L+L^{2}\right)^{d}\left(1-2 \cos \left(\frac{2 \pi}{2.5}\right) L+L^{2}\right)^{d} .
$$

Hence, generalized Gegenbauer processes encompass seasonal ARFIMA models. Figure 1 shows the power spectrum of a seasonal long memory process, $\left(1-L^{5}\right)^{0.4} h_{t}=\eta_{t-1}$, and a general Gegenbauer process, $(1-L)^{0.4}\left(1-2 \cos (2 \pi / 5) L+L^{2}\right)^{0.3}\left(1-2 \cos (2 \pi / 3) L+L^{2}\right)^{0.2} h_{t}=\eta_{t-1}$.

\section{$3 \quad$ Estimation and Forecasting}

\subsection{Whittle Likelihood Estimation of Short and Long Memory Parameters}

Following Zaffaroni (2009) and Asai, Chang, and McAleer (2017), we consider the log of the square of $y_{t}$ as $\underline{y}_{t}=\ln \left(y_{t}^{2}\right)$. By the transformation, we obtain the linearized model:

$$
\mathrm{y}_{t}=c+\alpha_{t}+u_{t}, \quad x_{t}=\mu+\alpha_{t}+v_{t}, \quad \alpha_{t}=[P(L)]^{-1}[\phi(L)]^{-1} \theta(L) \eta_{t-1},
$$

where $c=\mu+E\left(\ln \varepsilon_{t}^{2}\right), \alpha_{t}=h_{t}-\mu$, and $u_{t}=\ln \varepsilon_{t}^{2}-E\left(\ln \varepsilon_{t}^{2}\right)$. By Harvey, Ruiz, and Shephard (1994), it is known that $E\left(\ln \varepsilon_{t}^{2}\right)=-1.2703$ and $V\left(\ln \varepsilon_{t}^{2}\right)=\pi^{2} / 2$. Since $u_{t}$ is independent with mean zero and variance, $\sigma_{u}^{2}=\pi^{2} / 4, \mathrm{y}_{t}$ follows the long memory process with additive noise. Furthermore, we consider the mean subtracted series, $\boldsymbol{z}_{t}=\left(y_{t}^{\dagger}, x_{t}^{\dagger}\right)^{\prime}$, where $y_{t}^{\dagger}=\mathrm{y}_{t}-c$ and $x_{t}^{\dagger}=x_{t}-\mu$, in order to obtain:

$$
\boldsymbol{z}_{t}=\left(\begin{array}{c}
u_{t}+\sum_{j=0}^{\infty} \psi_{j} \eta_{t-j-1} \\
v_{t}+\sum_{j=0}^{\infty} \psi_{j} \eta_{t-j-1}
\end{array}\right)=\sum_{j=0}^{\infty} G_{j} \boldsymbol{e}_{t-j}
$$

where $\sum_{j=0}^{\infty} \psi_{j} z^{j}=[P(z)]^{-1}[\phi(z)]^{-1} \theta(z), \boldsymbol{e}_{t}=\left(u_{t}, v_{t}, \eta_{t}\right)^{\prime}$, and

$$
G_{0}=\left(\begin{array}{ccc}
1 & 0 & 0 \\
0 & 1 & 0
\end{array}\right), \quad G_{j}=\left(\begin{array}{ccc}
0 & 0 & \psi_{j} \\
0 & 0 & \psi_{j}
\end{array}\right) \quad(j \geq 1)
$$

with $E\left(\boldsymbol{e}_{t}\right)=0$ and $V\left(\boldsymbol{e}_{t}\right)=\Sigma_{e}=\operatorname{diag}\left(\sigma_{u}^{2}, \sigma_{v}^{2}, \sigma_{\eta}^{2}\right)$. Although the process $\left\{u_{t}\right\}$ is non-Gaussian, a reasonable estimation procedure is to maximize the quasi-likelihood, or the likelihood computed 
as if $\left\{u_{t}\right\}$ was Gaussian. Note that we estimate $\mu$ by the sample mean of $x_{t}$, to reduce the number of parameters.

Before applying the method of Zaffaroni (2009) and Asai, Chang, and McAleer (2017), we return to the estimation of a simple Gegenbauer ARMA process when $h_{t}$ is observed and $k=1$ and $d=0$. The asymptotic results of the ML estimator of Chung $(1994,1996)$ and Peiris and Asai (2016) indicate that the ML estimator of the location parameter, $\omega_{1}$, is $T$-consistent rather than $\sqrt{T}$-consistent, and that the estimator of $\omega_{1}$ and the remaining parameters are asymptotically independent. Since the WL estimator has the same limiting distribution as the QML estimator in the time domain (Taniguchi and Kakizawa, 2000, Chapter 5), it is reasonable to consider estimation of $\left(\omega_{1}, \ldots, \omega_{k}\right)$ and the remaining parameters separately in the RSV-GGLM model. We will explain in this section the semiparametric estimation technique of $\omega_{l}(l=1, \ldots, k)$ for $k$-factor Gegenbauer processes.

Define $\boldsymbol{\delta}=\left(d, d_{1}, \ldots, d_{k}, \phi_{1}, \ldots, \phi_{p}, \theta_{1}, \ldots, \theta_{q}, \sigma_{\eta}^{2}, \sigma_{v}^{2}\right)^{\prime}$ and $\boldsymbol{\omega}=\left(\omega_{1}, \ldots, \omega_{k}\right)^{\prime}$ as two vectors of parameters, where it is assumed $\left(\omega_{1}, \ldots, \omega_{k}\right)$ is known. By the specification, the process $\left\{\boldsymbol{z}_{t}\right\}$ in (6) is a second-order stationary process and has a spectral density matrix defined by $\boldsymbol{f}(\lambda)=$ $\frac{1}{2 \pi} \boldsymbol{k}(\lambda ; \boldsymbol{\delta}) \Sigma_{e} \boldsymbol{k}(\lambda ; \boldsymbol{\delta})^{*}$, where $\boldsymbol{k}(\lambda ; \delta)=\sum_{j=0}^{\infty} G_{j} e^{i \lambda j}$, which yields:

$$
\boldsymbol{f}(\lambda)=\frac{1}{2 \pi}\left(\begin{array}{cc}
K_{11}(\lambda) & K_{12}(\lambda) \\
K_{12}(\lambda)^{*} & K_{22}(\lambda)
\end{array}\right)
$$

with

$$
K_{11}(\lambda)=\sigma_{v}^{2}+\sigma_{\eta}^{2}\left|\psi\left(e^{i \lambda}\right)\right|^{2}, \quad K_{12}(\lambda)=\sigma_{\eta}^{2}\left|\psi\left(e^{i \lambda}\right)\right|^{2}, \quad K_{22}(\lambda)=\sigma_{u}^{2}+\sigma_{\eta}^{2}\left|\psi\left(e^{i \lambda}\right)\right|^{2} .
$$

Note that we can write $\left|\psi\left(e^{i \lambda}\right)\right|^{2}=\left|P\left(e^{i \lambda}\right)\right|^{-2} g_{h}(\lambda)$,

$$
\left|P\left(e^{i \lambda}\right)\right|^{2}=[2 \sin (\lambda / 2)]^{2 d} \prod_{l=1}^{k}\left[2\left(\cos (\lambda)-\cos \left(\omega_{l}\right)\right)\right]^{2 d_{l}},
$$

and $g_{h}(\lambda)$ is defined by equation (5). The $(1,1)$-element of $\boldsymbol{f}(\lambda)$ is the spectral density of $x_{t}$, which can be interpreted as the conventional signal plus noise process. The $(2,2)$-element of $\boldsymbol{f}(\lambda)$ is the spectral density of $\log y_{t}^{2}$, and corresponds to the result of Breidt, Crato, and de Lima (1998).

Let $\boldsymbol{I}_{T}(\boldsymbol{z}, \lambda)$ be the periodogram matrix defined by:

$$
\boldsymbol{I}_{T}(\boldsymbol{z}, \lambda)=\boldsymbol{w}_{T}(\lambda) \boldsymbol{w}_{T}(\lambda)^{*}, \quad-\pi<\lambda \leq \pi,
$$


where $\boldsymbol{w}_{T}(\lambda)$ is the finite Fourier transform, defined by:

$$
\boldsymbol{w}_{T}(\lambda)=\frac{1}{\sqrt{2 \pi}} \sum_{t=1}^{T} \boldsymbol{z}_{t} e^{i t \lambda} .
$$

For purposes of deriving the quasi-likelihood function, we treat the process $z_{t}$ as Gaussian. Choose the frequencies $\lambda_{j}, j=1, \ldots, n$, equi-spaced in the region $(-\pi, \pi]$ so that $\boldsymbol{f}(\lambda)$ is continuous at $\lambda=\lambda_{j}$ Then the finite Fourier transform $\boldsymbol{w}_{T}\left(\lambda_{j}\right), j=1, \ldots, n$, will have a complex-valued multivariate normal distribution which, for large $T$, is approximately independent, each with probability density function given by:

$$
\pi^{-1}\left\{\operatorname{det} \boldsymbol{f}\left(\lambda_{j} ; \boldsymbol{\delta}\right)\right\}^{-1 / 2} \exp \left[-\frac{1}{2} \operatorname{tr}\left\{\boldsymbol{f}^{-1}\left(\lambda_{j} ; \boldsymbol{\delta}\right) \boldsymbol{w}_{T}\left(\lambda_{j}\right) \boldsymbol{w}_{T}\left(\lambda_{j}\right)^{*}\right\}\right], \quad j=1, \ldots, T .
$$

As $\boldsymbol{w}_{T}\left(\lambda_{j}\right), j=1, \ldots, n$, constitutes a sufficient statistic for $\boldsymbol{\delta}$, an approximate log-likelihood function of $\boldsymbol{\delta}$ based on $\left\{\boldsymbol{z}_{1}, \ldots, \boldsymbol{z}_{T}\right\}$ is, excluding the constant term, given by:

$$
\bar{L}_{T}(\boldsymbol{\delta})=-\frac{1}{2} \sum_{j=1}^{T}\left[\log \operatorname{det} \boldsymbol{f}\left(\lambda_{j} ; \boldsymbol{\delta}\right)+\operatorname{tr}\left\{\boldsymbol{f}^{-1}\left(\lambda_{j} ; \boldsymbol{\delta}\right) \boldsymbol{I}_{T}\left(\boldsymbol{z}, \lambda_{j}\right)\right\}\right] .
$$

In integral form, equation (8) has the expression:

$$
-\frac{T}{4 \pi}\left[\int_{-\pi}^{\pi} \log \operatorname{det} \boldsymbol{f}(\lambda ; \boldsymbol{\delta}) d \lambda+\int_{-\pi}^{\pi} \operatorname{tr}\left\{\boldsymbol{f}^{-1}(\lambda ; \boldsymbol{\delta}) \boldsymbol{I}_{T}(\boldsymbol{z}, \lambda)\right\} d \lambda\right] .
$$

The function $\bar{L}_{T}(\boldsymbol{\delta})$ is called the quasi-log-likelihood function. The approximation was originally proposed by Whittle (1952) for scalar-valued stationary processes (see also Dunsmuir and Hannan (1976), and Taniguchi and Kakizawa (2000)). Define the Whittle likelihood (WL) estimator, $\hat{\boldsymbol{\delta}}_{T}$, which is obtained by minimizing $-\bar{L}_{T}(\boldsymbol{\delta})$. In practice, we use the discrete quasi-log-likelihood (8) with frequency $\lambda_{j}=2 \pi j / T(j=1, \ldots,\lfloor(T-1) / 2\rfloor)$, for the symmetry of the Fourier transform, as in standard empirical analysis.

Following Hosoya (1997), define the quantity:

$$
R_{j}(\boldsymbol{\delta})=H_{j}(\boldsymbol{\delta})+\int_{-\pi}^{\pi} \operatorname{tr}\left\{\boldsymbol{h}_{j}(\lambda, \boldsymbol{\delta}) \boldsymbol{f}(\lambda)\right\} d \lambda,
$$

where

$$
\begin{aligned}
& H_{j}(\boldsymbol{\delta})=\frac{\partial}{\partial \delta_{j}} \int_{-\pi}^{\pi} \log \operatorname{det} \boldsymbol{f}(\lambda ; \boldsymbol{\delta}) d \lambda \\
& \boldsymbol{h}_{j}(\lambda ; \boldsymbol{\delta})=\frac{\partial}{\partial \delta_{j}} \boldsymbol{f}^{-1}(\lambda ; \boldsymbol{\delta}) .
\end{aligned}
$$


Noting that

$$
\operatorname{det} \boldsymbol{f}(\lambda ; \boldsymbol{\delta})=\frac{1}{2 \pi}\left\{\sigma_{v}^{2} \sigma_{u}^{2}+\left(\sigma_{v}^{2} \sigma_{\eta}^{2}+\sigma_{u}^{2} \sigma_{\eta}^{2}\right)\left|\psi\left(e^{i \lambda}\right)\right|^{2}\right\},
$$

$R_{j}(\boldsymbol{\delta})$ is measurable with respect to $\boldsymbol{\delta}$ almost everywhere in $\lambda$. Denote $W$ as the matrix of derivatives, $W_{j l}=\partial R_{j} / \partial \delta_{l}$, evaluated at $\boldsymbol{\delta}=\boldsymbol{\delta}_{0}$.

As discussed in Asai, Chang, and McAleer (2017), we can obtain the asymptotic results of the WL estimator by checking the conditions of Hosoya (1997). If the vector of frequency parameters, $\boldsymbol{\omega}$, is known, we can apply the approach which was used to prove Theorem 2 in Chan and Tsai (2008) and Theorem 1 in Tsai, Rachinger, and Lin (2015), in order to verify Assumptions A, C, and D of Hosoya (1997) to show the consistency and asymptotic normality of the WL estimator. Then we obtain:

$$
\sqrt{T}\left(\hat{\boldsymbol{\delta}}_{T}-\boldsymbol{\delta}_{0}\right) \stackrel{d}{\longrightarrow} N\left(0, W^{-1} U\left(W^{*}\right)^{-1}\right),
$$

where $U$ is the matrix with $(j, l)$ th element represented as:

$$
\begin{aligned}
U_{j l}= & \left.4 \pi \int_{-\pi}^{\pi} \operatorname{tr}\left[\boldsymbol{h}_{j}\left(\lambda ; \delta_{0}\right) \boldsymbol{f}(\lambda) h_{l}\left(\lambda ; \delta_{0}\right) \boldsymbol{f}(\lambda)\right] d \lambda\right|_{\delta=\delta_{0}} \\
& +C_{1}\left\{\left[\left.\frac{1}{2 \pi} \int_{-\pi}^{\pi} k^{*}\left(\lambda_{1}\right) \boldsymbol{h}_{j}\left(\lambda_{1} ; \delta_{0}\right) k\left(\lambda_{1}\right)\right|_{\delta=\delta_{0}} d \lambda_{1}\right]_{11}\right\}^{2},
\end{aligned}
$$

and

$$
\left.\int_{-\pi}^{\pi} k^{*}(\lambda) \boldsymbol{h}_{j}\left(\lambda ; \delta_{0}\right) k(\lambda)\right|_{\delta=\delta_{0}} d \lambda=0 \quad \text { for } \delta_{j} \in\left(d, d_{1}, \ldots, d_{k}, \phi_{1}, \ldots, \phi_{p}, \theta_{1}, \ldots, \theta_{q}\right),
$$

with $C_{1}$ as the fourth cumulant of $u_{t}$, given by

$$
C_{1}=E\left(u_{t}^{4}\right)-3\left\{E\left(u_{t}^{2}\right)\right\}^{2}=\psi^{(3)}\left(\frac{1}{2}\right)-\frac{3 \pi^{2}}{16},
$$

where $\psi^{(3)}(z)$ is the penta gamma function (see equation (26.4.36) of Abramovits and Stegun (1970) for the result of the fourth moment of $u_{t}$ ). Although $U_{j l}$ defined by Theorem 2.2 in Hosoya (1997) is based on the fourth-order spectral density, it can be simplified as in (11) under Assumption F of Hosoya (1997) (see also equation (5.3.22) of Taniguchi and Kakizawa (2000) and Theorem 2 of Zaffaroni (2009)), which can be verified straightforwardly by the structure of the RSV-GGLM models.

We can allow a non-Gaussian distribution for $\varepsilon_{t}$ by setting $\sigma_{u}^{2}$ as a free parameter (see Harvey, Ruiz, and Shephard (1994), for instance), and by adding it in $\boldsymbol{\delta}$. As explained above, we use a 
two step procedure rather than estimating $(\boldsymbol{\delta}, \boldsymbol{\omega})$ simultaneously. In the first step, we obtain a consistent estimate of $\boldsymbol{\omega}, \hat{\boldsymbol{\omega}}$, by using a semiparametric method suggested by Hidalgo and Soulier (2004). In the second step, we obtain the WL estimate, $\hat{\boldsymbol{\delta}}$, by minimizing $-\bar{L}_{T}(\boldsymbol{\delta}, \hat{\boldsymbol{\omega}})$. Since we use $\hat{\boldsymbol{\omega}}$ instead of the true $\boldsymbol{\omega}$, no asymptotic results are yet available for this case.

\subsection{Semiparametric Estimation of Location Frequency Parameters and Iden- tification of $k$}

We explain the semiparametric technique of Hidalgo and Soulier (2004) for estimating the parameters of $\boldsymbol{\omega}$. We assume $k$ is known until we discuss the identification of parameters. For purposes of introducing the approach of Hidalgo and Soulier (2004), we consider a simple case of a univariate process which produces $I_{T}(\lambda)$, with the assumptions $d=0, \omega_{1} \neq 0, \omega_{2} \neq 0, d_{1} \geq d_{2}$, and $k=2$. Then we can estimate $\omega_{1}$ and $\omega_{2}$ consistently as:

$$
\hat{\omega}_{1}=\frac{2 \pi}{T} \arg \max _{1 \leq j \leq m} I_{T}\left(\lambda_{j}\right), \quad \hat{\omega}_{2}=\frac{2 \pi}{T} \arg \max _{\substack{1 \leq j \leq m \\\left|\lambda_{j}-\hat{\omega}_{1}\right| \geq z_{T} / T}} I_{T}\left(\lambda_{j}\right),
$$

where $z_{T}=T \exp (-\sqrt{\ln (T)})$, and $m$ is an integer between 1 and $\lfloor(T-1) / 2\rfloor$, satisfying at least:

$$
\frac{1}{m}+\frac{m}{T} \rightarrow 0 \text { as } T \rightarrow \infty
$$

After we estimate $\omega_{1}$, it is possible to estimate the second location parameter, $\omega_{2}$, which has a sufficient distance from the first location. For general $k$, we can estimate $\left(\omega_{1}, \ldots, \omega_{k}\right)$ sequentially.

Hidalgo and Soulier (2004) modified the GPH estimator of Geweke and Porter-Hudak (1983), which was originally suggested to estimate long memory parameter, $d$, using a log-periodogram regression, in order to estimate $d_{l}$ at the Gegenbauer frequency $\omega_{l}$. To identify the number of location frequencies, $k$, we follow the approach of Hidalgo and Soulier (2004), based on their modified GPH estimator for $d_{1}, \ldots, d_{k}$, which is defined by:

$$
\hat{d}_{l}=\sum_{\substack{1 \leq|j| \leq m \\ 0<\hat{\omega}_{l}+\lambda_{j} \leq \pi}} \xi_{k} \ln \left\{I_{T}\left(\hat{\omega}_{l}+\lambda_{j}\right)\right\}
$$

where $\xi_{k}=s_{m}^{-2}\left(\zeta\left(\lambda_{j}\right)-\bar{\zeta}_{m}\right), \zeta(\lambda)=-\ln \left(\left|1-e^{i \lambda}\right|\right), \bar{\zeta}_{m}=m^{-1} \sum_{j=1}^{m} \zeta\left(\lambda_{j}\right)$, and $s_{m}^{2}=\sum_{j=1}^{m}\left(\zeta\left(\lambda_{j}\right)-\right.$ $\left.\bar{\zeta}_{m}\right)^{2}$. Hidalgo and Soulier (2004) show that $m^{1 / 2}\left(\hat{d}_{l}-d_{l}\right)$ converges weakly to $N\left(0, \pi^{2} / 12\right)$, under 
the assumption of a Gaussian process. The procedure of Hidalgo and Soulier (2004) consists of the following steps: (i) Find the largest periodogram ordinate; (ii) if the corresponding estimate of $d_{l}$ is significant, add the respective Gegenbauer filter to the model, otherwise terminate the procedure; (iii) Exclude the neighborhood of the last pole from the periodogram, and repeat the procedure from (i) onward. For the assumption of Gaussianity of the procedure, we use the data of $x_{t}$, which produces $I_{T}\left(x_{t}, \lambda\right)$, excluding $\mathrm{y}_{t}$.

\subsection{Estimating and Forecasting Volatility}

Using the WL estimates above, we can obtain the minimum mean square linear estimator (MMSLE) of $h_{t}$ from the work of Harvey (1998) and Asai, Chang, and McAleer (2017). Define $\boldsymbol{x}^{\dagger}=\left(x_{1}^{\dagger}, \ldots, x_{T}^{\dagger}\right)^{\prime}, \boldsymbol{y}^{\dagger}=\left(y_{1}^{\dagger}, \ldots, y_{T}^{\dagger}\right)^{\prime}, \boldsymbol{h}=\left(h_{1}, \ldots, h_{T}\right)^{\prime},, \boldsymbol{v}=\left(v_{1}, \ldots, v_{T}\right)^{\prime}$, and $\boldsymbol{u}=\left(u_{1}, \ldots, u_{T}\right)^{\prime}$ in order to obtain:

$$
\boldsymbol{x}^{\dagger}=\boldsymbol{h}-\mu \mathbf{1}_{T}+\boldsymbol{v}, \quad \boldsymbol{y}^{\dagger}=\boldsymbol{h}-\mu \mathbf{1}_{T}+\boldsymbol{u},
$$

where $\mathbf{1}_{T}$ is an $T \times 1$ vector of ones. Then, the minimum mean square linear estimator of $\boldsymbol{h}$ is given by:

$$
\tilde{\boldsymbol{h}}=\mu \mathbf{1}_{T}+\tau^{-1}\left(I_{T}-\Sigma_{\tau}^{-1}\right)\left(\sigma_{v}^{-2} \boldsymbol{x}^{\dagger}+\sigma_{u}^{-2} \boldsymbol{y}^{\dagger}\right),
$$

where $\tau=\sigma_{v}^{-2}+\sigma_{u}^{-2}, \Sigma_{\tau}=I_{T}+\tau \Sigma_{h}$, and $V(\boldsymbol{h})=\Sigma_{h}$. We obtain $\Sigma_{h}$ via the algorithm of McElroy and Holan (2012) (see the Appendix for details). Harvey (1998) recommends using the volatility estimate:

$$
\tilde{\sigma}_{t}^{2}=\tilde{\sigma}_{\tilde{y}}^{2} \exp \left(\tilde{h}_{t}\right)
$$

where $\tilde{\sigma}_{\tilde{y}}^{2}=T^{-1} \sum_{t=1}^{n} \tilde{y}_{t}^{2}$, and $\tilde{y}_{t}=y_{t} \exp \left(-0.5 \tilde{h}_{t}\right)$ are the heteroskedasticity-corrected observations.

For predicting the observations for $x_{t}^{\dagger}$ and $y_{t}^{\dagger}$ for $t=T+1, \ldots, T+l$, denote $\boldsymbol{x}_{l}^{\dagger}$ and $\boldsymbol{y}_{l}^{\dagger}$ as the $l \times 1$ vectors of predicted values, respectively. Then the corresponding MMSLEs are given by:

$$
\tilde{\boldsymbol{x}}_{l}^{\dagger}=\boldsymbol{R}_{x} \Sigma_{x}^{-1} \boldsymbol{x}^{\dagger}, \quad \tilde{\boldsymbol{y}}_{l}^{\dagger}=\boldsymbol{R}_{y} \Sigma_{y}^{-1} \boldsymbol{y}^{\dagger}
$$

where $\Sigma_{x}=\Sigma_{h}+\sigma_{v}^{2} I_{T}, \Sigma_{y}=\Sigma_{h}+\sigma_{u}^{2} I_{T}, R_{x}\left(R_{y}\right)$ is the $l \times T$ matrix of covariances between $\boldsymbol{x}_{l}^{\dagger}$ and $\boldsymbol{x}^{\dagger}\left(\boldsymbol{y}_{l}^{\dagger}\right.$ and $\left.\boldsymbol{y}^{\dagger}\right)$. Using $\tilde{\boldsymbol{h}}_{l}=\mu \mathbf{1}_{l}+\tau^{-1}\left(\sigma_{v}^{-2} \tilde{\boldsymbol{x}}_{l}^{\dagger}+\sigma_{u}^{-2} \tilde{\boldsymbol{y}}_{l}^{\dagger}\right)$, the predictions of $\sigma_{T+j}^{2}(j=1, \ldots, l)$ are given by exponentiating the elements of $\tilde{\boldsymbol{h}}_{l}$, and multiplying by $\tilde{\sigma}_{\tilde{y}}^{2}$. 


\subsection{Finite Sample Properties}

We conducted Monte Carlo experiments for investigating the finite sample properties of the WL estimator of $\boldsymbol{\delta}$ and the semiparametric estimator of $\boldsymbol{\omega}$. We consider two kinds of long memory components:

$$
\left(d_{0}, d_{1}, d_{2}, \omega_{1}, \omega_{2}\right)= \begin{cases}(0.4,0.4,0.4,2 \pi / 5,2 \pi / 2.5) & \text { for Seasonal Long Memory } \\ (0.4,0.3,0.2,2 \pi / 5,2 \pi / 3) & \text { for General Gegenbauer Long Memory }\end{cases}
$$

for which the power spectra are shown in Figure 1. Note that the original specification of the RSV-SLM (Seasonal Long Memory) model is given by equations (1)-(3), with $P(L)=\left(1-L^{s}\right)^{d}$, and $s=5$ corresponds to the above DGP. For the remaining parameters, we specify $\left(\sigma_{v}, \sigma_{\eta}, \phi, \mu\right)=$ $(0.2,0.4,0.6,-0.1)$. We consider sample sizes $T=\{1024,2048\}$, with $R=5000$ replications.

The first experiment considers selection of the number of location parameters, $k$. Table 1(a) shows the relative frequencies for selecting the number of long memory parameters via the procedure of Hidalgo and Soulier (2004), with $m=0.5 T^{0.7}$. The mean selected value indicates that there is an upward bias in the procedure for the sample sizes, which may be caused by over-rejection of the modified GPH estimator. Table 1(b) presents the relative frequencies of containing the true location parameters, such that $\left|\hat{\omega}_{j}-\omega_{j}\right|<z_{T} / T=\exp (-\sqrt{\ln (T)})$ for each selected value of $k$. The frequencies of selecting true values increase as the sample size and/or the true value of long memory parameter increases. As a result, the approach of Hidalgo and Soulier (2004) tends to select larger values of $k$ for $T=2048$, but the location parameter estimates chosen by the approach tend to include the true parameters.

The second experiment examines the finite sample properties of the WL estimator under the true values of $\boldsymbol{\omega}$. Table 2 reports the sample mean, standard deviation, and root mean squared error (RMSE) of the WL estimator of $\boldsymbol{\delta}$. For $\sigma_{u}, \phi, d, d_{1}$, and $d_{2}$, the bias of the estimator is negligible for both $T=1024$ and $T=2048$. While the bias for $\sigma_{v}$ is upward, that of $\sigma_{\eta}$ is downward. Compared with the case $T=1024$, there is no improvement in the biases of $\sigma_{v}$ and $\sigma_{\eta}$. However, the results for $T=2048$ have smaller standard deviations and RMSEs. Table 2 also shows the sample mean, standard deviation, and root mean squared error of the estimator of $\mu$ by the sample mean of $x_{t}$, with the same implications.

By the structure of the RSV-GGLM model, we consider the quasi-likelihood ratio (QLR) 
statistic for testing the RSV-SLM model against the RSV-GGLM model. As shown in Theorem 3.1.3 of Taniguchi and Kakizawa (2000) in the general framework, the QLR test under known $\boldsymbol{\omega}$ has the asymptotic $\chi^{2}(2)$ distribution. The last entries of Table 2 report the rejection frequencies of the QLR statistic at the five percent significance level, indicating that the rejection frequency under the null model approaches the nominal size of $5 \%$ as $T$ increases. Under the alternative model, the sample size of $T=1024$ is sufficient to reject the null hypothesis for the parameter set.

\section{Empirical Analysis}

The empirical analysis focuses on estimating and forecasting the RSV-GGLM model for three sets of stock indices, namely Standard \& Poors 500 (S\&P), FTSE 100 (FTSE), and Nikkei 225 (Nikkei). For each return computed for 1-min intervals of the trading day at $t$ between 9:30 a.m. and 4:00 p.m., we calculated the daily volatility using the realized kernel (RK) estimator of Barndorff-Nielsen et al. (2008), which is consistent and robust to microstructure noise and jumps. We also calculate the corresponding returns for the three assets.

We denote the return and $\log$ of the RK estimate at day $t$ as $r_{t}$ and $x_{t}$, respectively. The sample period is from March 23, 2007 to September 19, 2017, to obtain the last 2548 observations, excluding holidays and weekends. We use the first $T=2048$ returns for estimating the RSVGGLM models, and the remaining 500 series for forecasting. The estimation period includes the Global Financial Crisis from 2007-2009.

Table 3 presents the descriptive statistics of the returns and log-volatility for the whole sample. The empirical distribution of the returns is highly leptokurtic, and is skewed to the left. Compared with the returns series, the distribution of log-volatility is closer to the normal distribution, but is skewed to the right, and the kurtosis exceeds three. As our interest is on volatility, we use the mean subtracted returns, $y_{t}=r_{t}-\bar{r}$. Figure 2 shows the sample spectral density for log-volatility. There is a clear evidence that the spectral density is unbounded at the origin, $\lambda=0$. Since there are several peaks apart from the origin, it is worth investigating the general pattern for the structure of long memory.

Table 4 gives the semiparametric estimates of the location parameter $\boldsymbol{\omega}$, accompanied by 
the results of the procedure of Hidalgo and Soulier (2004) for selecting the number $k$. While $k=2$ was selected for $\mathrm{S} \& \mathrm{P}$ and FTSE, the procedure chose $k=3$ for Nikkei. In the following analysis, we set $\hat{\omega}_{0}=0$ from Figure 2 . Table 4 shows that the periods of frequencies are close to $(20,10,5)$ for FTSE, implying that $P(L)=\left(1-L^{20}\right)^{d}$ is another candidate for specifying the long memory structure. As an alternative specification, we also consider $P(L)=\left(1-L^{30}\right)^{d}$ and $P(L)=\left(1-L^{20}\right)^{d}$ for S\&P and Nikkei, respectively.

Table 5 gives the WL estimates for the RSV-GGLM and RSV-SLM models. While the QLR test rejected the null hypothesis of the RSV-SLM model for S\&P and Nikkei, it failed to reject the null hypothesis for FTSE. For S\&P, the estimate of $d$ is close to 0.5 , which is dominant compared with other estimates of long memory parameters, $d_{l}(l=1,2,3)$. All the estimates of the long memory parameters are significant at five percent level, rejecting the RSV model with the $\operatorname{ARFIMA}(1, d, 0)$ specification. The estimate of $\sigma_{u}$ is close to $\pi / \sqrt{2}$, which is obtained by the standard normal distribution for $\varepsilon_{t}$. The estimates of the RSV-SLM model for FTSE indicate that the estimate of $d$ is 0.056 , and is significant. Since the estimate of $d$ in the unrestricted RSVGGLM model is 0.382 , the value of long memory parameter becomes smaller, and the estimate of $\phi$ becomes close to one in the RSV-SLM model, in order to capture the effect of the mass close to the origin in Figure 2(b). The estimation results for Nikkei 225 are similar to those of S\&P.

We examine the performance of the out-of-sample forecasts using the root mean squared error (RMSE) and the Diebold and Mariano (1995) test for equal forecast accuracy. The benchmark model is the HAR model of Corsi (2009), which is given by:

$$
x_{t}=c+\phi_{d} x_{t-1}+\phi_{w}\left(x_{t-1}\right)_{5}+\phi_{w}\left(x_{t-1}\right)_{20}+\text { error }
$$

where $\left(x_{t-1}\right)_{h}$ denotes the $h$-horizon average of past $x_{t}$. Note that $\left(x_{t-1}\right)_{5}$ and $\left(x_{t-1}\right)_{22}$ are the weekly and monthly averages, respectively. The model is interpreted as the $\operatorname{AR}(22)$ process with the parameter restrictions. Although the model is not technically a long memory process, it approximates the effects of longer horizons in a simple and parsimonious way. We use $x_{T+j}$ $(j=1, \ldots, F)$ as the proxy of the true log-volatility. Fixing the sample size at 2048 for the rolling window, we re-estimated the model and computed the one step ahead forecasts of log-volatility 
for the last $F=500$ days. RMSE is defined as:

$$
\sqrt{\frac{1}{F} \sum_{j=1}^{F}\left(\tilde{h}_{T+j}-x_{T+j}\right)^{2}},
$$

where $\tilde{h}_{T+l}$ is the forecast of $h_{T+j}$ for the RSV models, and that of $x_{T+j}$ for the HAR model. As above, we select the optimal $k$ each time for estimating the RSV-GGLM model. As an ad hoc approach, we also consider a combined forecast obtained by the weighted average of the forecasts of RSV-GGLM and RSV-SLM models, with weights $(-1,2)$.

Table 6 also indicates the HAR model has the largest RMSEs. The RSV-SLM model provides smaller RMSEs than the RSV-GGLM model, while the combined forecast gives the smallest values. The Diebold-Mariano test against the forecast of the HAR model are rejected at the five percent significance level in all cases.

The empirical results show that the data for S\&P, FTSE, and Nikkei prefer the more flexible structure for long memory in log-volatility than the simple ARFIMA process. For sample data, S\&P and Nikkei favor the RSV-GGLM model, while FTSE selected the RSV-SLM model. The results of the out-of-sample forecasts indicate that the RSV-SLM model gives better forecasts than the RSV-GGLM model. However, the forecasts can be improved by combining the RSV-GGLM and RSV-SLM models.

\section{Concluding Remarks}

In this paper, we considered a new realized stochastic volatility model with general Gegenbauer long memory (RSV-GGLM), which encompasses the new RSV model with seasonal long memory (RSV-SLM). We suggested a two-step estimator, in which the first step estimator gives the estimates of the location parameters of the Gegenbauer frequencies, which converges faster than the speed of $T^{1 / 2}$. The second step uses the Whittle likelihood (WL) estimation method, for which the asymptotic distribution is the same as that of the quasi-maximum likelihood estimator when the location parameter is known. Then we conducted Monte Carlo experiments for investigating the finite sample properties of both estimators, and found that the first step estimator works satisfactorily, and that the finite sample bias for the WL estimator is negligible for $T=2048$. 
The estimation results for S\&P, FTSE, and Nikkei indicate that the simple ARFIMA process for log-volatility is rejected, favoring either of the RSV-GGLM and RSV-SLM models. The forecasting results indicate that combining the forecasts of both models gives improved forecasts compared with the original ones. These results indicate that RSV models with general long memory are useful additions to the existing models in the literature.

\section{Appendix}

We explain the calculation of the coefficients of the $\mathrm{MA}(\infty)$ representation of the general Gegenbauer process in equation (3), and the calculation of the autocovariance functions.

Even for the simple Gegenbauer process with ARMA parameters, it is not easy to obtain explicit formulas for the coefficients for the $\mathrm{MA}(\infty)$ representation and the autocovariances that are valid for all lags. Recently, McElroy and Holan (2012, 2016) developed a computationally efficient method for calculating these values. The spectral density of the general Gegenbauer process, $h_{t}$, can be written as:

$$
f_{h}(\lambda)=\frac{\sigma_{\eta}^{2}}{2 \pi} g_{h}(\lambda)[2 \sin (\lambda / 2)]^{-2 d} \prod_{l=1}^{k}\left[2\left(\cos (\lambda)-\cos \left(\omega_{l}\right)\right)\right]^{-2 d_{l}}, \quad-\pi<\lambda<\pi,
$$

where $g_{h}(\omega)$ is defined by (5). For convenience, we define $\kappa(z)$ so that $g(\lambda)=\left|\kappa\left(e^{-i \lambda}\right)\right|^{2}$. Then, $\kappa(z)$ takes the form $\kappa(z)=\prod_{l}\left(1-\zeta_{l} z\right)^{p_{l}}$ for (possibly complex) reciprocal roots, $\zeta_{l}$, of the moving average and autoregressive polynomials, where $p_{l}$ is one if $l$ corresponds to a moving average root, and minus one if $l$ corresponds to an autoregressive root. We assume $d>\max \left\{d_{l}\right\}$, as suggested by the empirical results in Section 4 .

Define:

$$
\begin{aligned}
g_{j} & =2 \sum_{l} \frac{p_{l} \zeta_{l}^{j}}{j}, \\
\beta_{j} & =\frac{2}{j}\left\{d+2 \sum_{l=1}^{k} d_{l} \cos \left(\omega_{l} j\right)\right\}+g_{j}, \\
\tilde{\psi}_{j} & =\frac{1}{2 j} \sum_{m=1}^{l} m \beta_{m} \tilde{\psi}_{j-m}, \quad \tilde{\psi}_{0}=1 .
\end{aligned}
$$


McElroy and Holan (2012) showed that the $\mathrm{MA}(\infty)$ representation of (3) is given by:

$$
h_{t+1}=\mu+\sum_{j=0}^{\infty} \tilde{\psi}_{j} \eta_{t-j},
$$

and the autocovariances of $h_{t}$ for $l \geq 0$ are given by:

$$
\gamma_{l}=\sigma^{2} \sum_{j=0}^{J-1} \tilde{\psi}_{j} \tilde{\psi}_{j+l}+R_{J}(l),
$$

where

$$
R_{J}(l)=\sigma^{2}\left\{J^{-1+2 d} \frac{F(1-d, 1-2 d ; 2-2 d ;-l / J)}{\Gamma^{2}(d)(1-2 d)}\right\}\{1+o(1)\},
$$

and $F(a, b ; c ; z)$ is the hypergeometric function evaluated at $z$. Note that $\gamma_{-l}=\gamma_{l}$. McElroy and Holan (2012) recommend using the cutoff value $J \geq 2,000$. We set $J=20 T$ with $T=\{1024,2048\}$ in this paper. 


\section{References}

Abramovits, M. and N. Stegun (1970), Handbook of Mathematical Functions, Dover Publications, N.Y.

Andersen, T.G., T. Bollerslev, F.X. Diebold, and P. Labys (2001), "The Distribution of Realized Exchange Rate Volatility", Journal of the American Statistical Association, 96, 42-55.

Andersen, T.G., T. Bollerslev, F.X. Diebold, and P. Labys (2003), "Modeling and Forecasting Realized Volatility", Econometrica, 71, 529-626.

Artiach, M. and J. Arteche (2012), "Doubly Fractional Models for Dynamic Heteroscedastic Cycle", Computational Statistics \& Data Analysis, 56, 2139-2158.

Asai, M., C.-L. Chang, and M. McAleer (2017), "Realized stochastic volatility with general asymmetry and long memory", Journal of Econometrics, 199, 202-212.

Asai, M., M. McAleer, and M.C. Medeiros (2012), "Asymmetry and Long Memory in Volatility Modeling", Journal of Financial Econometrics, 10, 495-512.

Baillie R.T., T. Bollerslev, and H.O. Mikkelsen (1996). "Fractionally Integrated Generalized Autoregressive Conditional Heteroskedasticity", Journal of Econometrics, 74, 3-30.

Bollerslev, T. and H.O. Mikkelsen (1996), "Modeling and Pricing Long-Memory in Stock Market Volatility", Journal of Econometrics, 73, 151-184.

Bordignon, S., M. Caporin, and F. Lisi (2007), "Generalised Long-Memory GARCH Models for Intra-Daily Volatility", Computational Statistics \& Data Analysis, 51, 5900-5912.

Bordignon, S., M. Caporin, and F. Lisi (2009), "Periodic Long-Memory GARCH Models", Econometric Reviews, 28, 60-82.

Breidt, F.J., N. Crato, and P. de Lima (1998), "The Detection and Estimation of Long Memory", Journal of Econometrics, 83, 325-348.

Chan, K.S. and H. Tsai (2012), "Inference of seasonal long-memory aggregate time series", Bernoulli, 18, $1448-1464$.

Chung, C.F. (1996a), "Estimating A Generalized Long Memory Process", Journal of Econometrics, 73, $237-259$.

Chung, C.F. (1996b), "A generalized fractionally integrated autoregressive moving-average process", Journal of Time Series Analysis, 17, 111-140.

Corsi, F. (2009), "A Simple Approximate Long-Memory Model of Realized Volatility", Journal of Financial Econometrics, 7, 174-196.

Diebold, F. and R. Mariano (1995), "Comparing Predictive Accuracy", Journal of Business and Economic Statistics, 13, 253-263.

Dunsmuir, W. and E.J. Hannan (1976), "Vector Linear Time Series Models", Advances in Applied Probability, 8, 339-364.

Gray, H.L., N. Zhang, and W.A. Woodward (1989), "On Generalized Fractional Processes", Journal of Time Series Analysis, 10, 233-257.

Geweke, J. and S. Porter-Hudak (1983), "The Estimation and Application of Long-Memory Time Series Models", Journal of Time Series Analysis, 4, 87-104.

Hansen, P.R. and Z. Huang (2016), "Exponential GARCH Modeling with Realized Measures of Volatility", Journal of Business 83 Economic Statistics, 34, 269-287. 
Hansen, P.R., Z. Huang, and H.H. Shek (2012), "Realized GARCH: A Complete Model of Returns and Realized Measures of Volatility", Journal of Applied Econometrics, 27, 877-906.

Harvey, A. (1998), "Long Memory in Stochastic Volatility", in: Knight, J. and S. Satchell (eds.), Forecasting Volatility in Financial Markets, Oxford: Butterworth-Haineman, 307-320.

Harvey, A.C., E. Ruiz, and N. Shephard (1994), "Multivariate Stochastic Variance Models", Review of Economic Studies, 61, 247-264.

Hidalgo, J. and P. Soulier (2004), "Estimation of the location and exponent of the spectral singularity of a long memory process", Journal of Time Series Analysis, 25, 55-81.

Hosoya, Y. (1997), "A Limit Theory for Long-Range Dependence and Statistical Inference on Related Models", Annals of Statistics, 25, 105-137.

Koopman, S.J., B. Jungbacker, and E. Hol (2005), "Forecasting Daily Variablity of the S\&P 100 Stock Index Using Historical Realized and Implied Volatility Measurements", Journal of Empirical Finance, 12, 445-475.

Koopman, S.J. and M. Scharth (2013), "The Analysis of Stochastic Volatility in the Presence of Daily Realized Measures", Journal of Financial Econometrics, 11, 76-115.

McElroy, T. S. and S. H. Holan (2012), "On the Computation of Autocovariances for Generalized Gegenbauer Processes", Statistica Sinica, 22, 1661-1687.

McElroy, T. S. and S. H. Holan (2016), "Computation of the Autocovariances for Time Series with Multiple Long-Range Persistencies", Computational Statistics \& Data Analysis, 101, 44-56.

Peiris, S. and M. Asai (2016), "Generalized Fractional Processes with Long Memory and Time Dependent Volatility Revisited", Econometrics, 4(3), 121.

Pong S., M.B. Shackelton, S.J. Taylor, and X. Xu (2004), "Forecasting currency volatility: a comparison of implied volatilities and AR(FI)MA models", Journal of Banking and Finance, 28, 2541-2563.

Porter-Hudak, S. (1990), "An Application of the Seasonal Fractionally Differenced Model to the Monetary Aggregates", Journal of the American Statistical Association, 85, 338-344

Shirota, S., T. Hizu, and Y. Omori (2014), "Realized Stochastic volatility with Leverage and Long Memory", Computational Statistics \& Data Analysis, 76, 618-641.

Takahashi, M., Y. Omori, and T. Watanabe (2009), "Estimating Stochastic Volatility Models Using Daily Returns and Realized Volatility Simultaneously", Computational Statistics \& Data Analysis, 53, $2404-2426$.

Taniguchi, M. and Y. Kakizawa (2000), Asymptotic Theory of Statistical Inference for Time Series, New York: Springer-Verlag.

Tsai, H., H. Rachinger, and E.M.H. Lin (2015), "Inference of Seasonal Long-Memory Time Series with Measurement Error", Scandinavian Journal of Statistics, 42, 137-154.

Whittle, P. (1952), "Some Results in Time Series Analysis", Skandivanisk Aktuarietidskrift, 35, 48-60.

Woodward, W.A., Q.C. Cheng, and H.L. Gray (1998), "A k-Factor GARMA Long Memory Model", Journal of Time Series Analysis, 19, 485-504.

Zaffaroni, P. (2009), "Whittle Estimation of EGARCH and Other Exponential Volatility Models", Journal of Econometrics, 151, 190-200. 
Table 1: Finite Sample Performance of Selection Procedures

(a) Relative Frequencies of Selecting $k$

\begin{tabular}{c|cccc}
\hline \hline \multirow{2}{*}{$k$} & \multicolumn{2}{|c}{ RSV-SLM } & \multicolumn{2}{c}{ RSV-GGLM } \\
\cline { 2 - 5 } & $T=1024$ & $T=2048$ & $T=1024$ & $T=2048$ \\
\hline 0 & 0.0000 & 0.0000 & 0.0000 & 0.0000 \\
1 & 0.0114 & 0.0000 & 0.3892 & 0.0002 \\
2 & 0.6032 & 0.2354 & 0.3058 & 0.2984 \\
3 & 0.2418 & 0.0870 & 0.0384 & 0.0236 \\
4 & 0.1436 & 0.3910 & 0.2480 & 0.2030 \\
5 & 0.0000 & 0.2866 & 0.0186 & 0.4276 \\
6 & 0.0000 & 0.0000 & 0.0000 & 0.0382 \\
Mean & 2.5176 & 3.7288 & 2.2010 & 3.8740 \\
\hline \hline
\end{tabular}

Note: The entries show the relative frequencies of selected values of $k$ by the procedure of Hidalgo and Soulier (2004).

(b) Relative Frequencies of Containing True Location Parameters

\begin{tabular}{c|cccc}
\hline \hline \multirow{2}{*}{ Param. } & \multicolumn{2}{|c}{ RSV-SLM } & \multicolumn{2}{c}{ RSV-GGLM } \\
\cline { 2 - 5 } & $T=1024$ & $T=2048$ & $T=1024$ & $T=2048$ \\
\hline$\omega_{1}$ & 0.9990 & 1.0000 & 0.9858 & 0.9972 \\
$\omega_{2}$ & 0.9798 & 0.9988 & 0.0730 & 0.3320 \\
\hline
\end{tabular}

Note: The entries show the relative frequencies of containing true location parameters such that $\left|\hat{\omega}_{j}-\omega_{j}\right|<\exp (-\sqrt{\ln (T)})$ for each selected value of $k$. 
Table 2: Finite Sample Performance of WL Estimator for RSV-GGLM Models

\begin{tabular}{|c|c|c|c|c|c|c|}
\hline \multirow[b]{2}{*}{ Parameters } & \multicolumn{3}{|c|}{$\overline{\text { DGP: RSV-SLM }}$} & \multicolumn{3}{|c|}{ DGP: RSV-GGLM } \\
\hline & True & $T=1024$ & $T=2048$ & True & $T=1024$ & $T=2048$ \\
\hline \multirow[t]{3}{*}{$\sigma_{u}$} & 2.2214 & 2.2192 & 2.2192 & 2.2214 & 2.2195 & 2.2195 \\
\hline & & $(0.0846)$ & $(0.0605)$ & & $(0.0846)$ & $(0.0605)$ \\
\hline & & {$[0.0847]$} & {$[0.0606]$} & & {$[0.0847]$} & [0.0605] \\
\hline \multirow[t]{3}{*}{$\sigma_{v}$} & 0.02 & 0.0352 & 0.0375 & 0.02 & 0.0298 & 0.0294 \\
\hline & & $(0.0407)$ & $(0.0377)$ & & $(0.0323)$ & $(0.0285)$ \\
\hline & & {$[0.0434]$} & {$[0.0416]$} & & [0.0337] & {$[0.0300]$} \\
\hline \multirow[t]{3}{*}{$\sigma_{\eta}$} & 0.4 & 0.3063 & 0.3032 & 0.4 & 0.3047 & 0.3025 \\
\hline & & $(0.0236)$ & $(0.0164)$ & & $(0.0228)$ & $(0.0159)$ \\
\hline & & {$[0.0966]$} & {$[0.0982]$} & & {$[0.0980]$} & {$[0.0988]$} \\
\hline \multirow[t]{3}{*}{$\phi$} & 0.6 & 0.6318 & 0.6226 & 0.6 & 0.6190 & 0.6110 \\
\hline & & $(0.0828)$ & $(0.0631)$ & & $(0.0861)$ & $(0.0661)$ \\
\hline & & {$[0.0887]$} & {$[0.0670]$} & & {$[0.0881]$} & {$[0.0670]$} \\
\hline \multirow[t]{3}{*}{$d$} & 0.4 & 0.3808 & 0.3897 & 0.4 & 0.3843 & 0.3945 \\
\hline & & $(0.0927)$ & $(0.0647)$ & & $(0.0920)$ & $(0.0661)$ \\
\hline & & {$[0.0947]$} & {$[0.0655]$} & & {$[0.0933]$} & {$[0.0663]$} \\
\hline \multirow[t]{3}{*}{$d_{1}$} & 0.4 & 0.4235 & 0.4221 & 0.3 & 0.3128 & 0.3120 \\
\hline & & $(0.0353)$ & $(0.0277)$ & & $(0.0319)$ & $(0.0240)$ \\
\hline & & {$[0.0424]$} & {$[0.0355]$} & & {$[0.0344]$} & {$[0.0268]$} \\
\hline \multirow[t]{3}{*}{$d_{2}$} & 0.4 & 0.4172 & 0.4118 & 0.2 & 0.2031 & 0.2039 \\
\hline & & $(0.0328)$ & $(0.0222)$ & & $(0.0310)$ & $(0.0220)$ \\
\hline & & {$[0.0370]$} & {$[0.0252]$} & & {$[0.0312]$} & {$[0.0224]$} \\
\hline \multirow[t]{3}{*}{$\mu$} & -0.1 & -0.1010 & -0.1010 & -0.1 & -0.1081 & -0.1006 \\
\hline & & $(0.3673)$ & $(0.3386)$ & & $(0.5085)$ & $(0.4692)$ \\
\hline & & {$[0.3673]$} & {$[0.3386]$} & & {$[0.5085]$} & {$[0.4692]$} \\
\hline QLR Test & & 0.0806 & 0.0510 & & 1.0000 & 1.0000 \\
\hline
\end{tabular}

Note: Except for ' $\mu$ ' and 'LR test', entries show the means of the WL estimates under true $\boldsymbol{\omega}$, and $\mu$ is estimated by the sample mean of $x_{t}$. Standard errors are in parentheses, and root mean squared errors are in brackets. 'QLR Test' reports the rejection frequencies of the QLR statistic for testing the null hypothesis of the RSV-SLM model. The critical value of the QLR test with true $\boldsymbol{\omega}$ is given by 5.9915, which is the upper five percentile of $\chi^{2}(2)$ distribution. 
Table 3: Descriptive Statistics of Return and Log-Volatility

\begin{tabular}{c|cccc}
\hline \hline Data & Average & Std. Dev. & Skewness & Kurtosis \\
\hline Return & & & & \\
S\&P & 0.0118 & 0.0315 & 13.281 & 311.00 \\
FTSE & 0.0080 & 0.0154 & 9.0698 & 133.79 \\
Nikkei & 0.0109 & 0.0229 & 7.9595 & 87.938 \\
Log-Volatility & & & \\
S\&P & -5.2585 & 1.2314 & 0.4536 & 3.2287 \\
FTSE & -5.3742 & 0.9748 & 0.5915 & 3.4626 \\
Nikkei & -5.1325 & 0.9613 & 0.6329 & 3.9309 \\
\hline \hline
\end{tabular}

Table 4: Semiparametric Estimates of Location Parameters

\begin{tabular}{c|ccc|ccc|ccc}
\hline \hline & \multicolumn{3}{|c|}{ S\&P } & \multicolumn{3}{c|}{ FTSE } & \multicolumn{3}{c}{ Nikkei } \\
$l$ & $\hat{\omega}_{l}$ & Days & $P$-value & $\hat{\omega}_{l}$ & Days & $P$-value & $\hat{\omega}_{l}$ & Days & $P$-value \\
\hline 0 & 0.0010 & - & $0.0012^{*}$ & 0.0010 & - & $0.0001^{*}$ & 0.0020 & - & $0.0002^{*}$ \\
1 & 0.0674 & 29.681 & $0.0017^{*}$ & 0.0947 & 21.113 & $0.0001^{*}$ & 0.1006 & 19.884 & $0.0004^{*}$ \\
2 & 0.1416 & 14.124 & $0.0031^{*}$ & 0.2002 & 9.9902 & $0.0006^{*}$ & 0.2041 & 9.7990 & $0.0015^{*}$ \\
3 & 0.4502 & 4.4425 & 0.2054 & 0.4131 & 4.8416 & 0.0888 & 0.2744 & 7.2883 & $0.0050^{*}$ \\
4 & & & & & & & 0.6973 & 2.8683 & 0.1947 \\
\hline \hline
\end{tabular}

Note: The estimates of $\omega_{l}$ are reported with the unit of $\pi$. 'Days' indicates the period corresponding to $\hat{\omega}_{l}$. ' $P$-value' shows the $P$-value for the modified GPH estimates of $d_{l}$, and '*' indicates the significance at the five percent level. 
Table 5: WL Estimates of RSV-GGLM and RSV-SLM Models

\begin{tabular}{c|cccccc}
\hline \hline \multirow{2}{*}{ Parameters } & \multicolumn{2}{|c}{ S\&P } & \multicolumn{2}{c}{ FTSE } & \multicolumn{2}{c}{ Nikkei } \\
& RSV-GGLM & RSV-SLM & RSV-GGLM & RSV-SLM & RSV-GGLM & RSV-SLM \\
\hline$\sigma_{u}$ & 2.0415 & 2.2103 & 2.1159 & 2.0325 & 2.0274 & 2.0293 \\
& $(0.0369)$ & $(0.0651)$ & $(0.0871)$ & $(0.0453)$ & $(0.1733)$ & $(0.1036)$ \\
$\sigma_{v}$ & 0.5414 & $1.4024 \times 10^{-4}$ & 0.0195 & 0.3962 & 0.4303 & 0.4237 \\
& $(0.0132)$ & $\left(0.0934 \times 10^{-4}\right)$ & $(0.0005)$ & $(0.0062)$ & $(0.0094)$ & $(0.0345)$ \\
$\sigma_{\eta}$ & 0.1110 & 0.6727 & 0.3825 & 0.1849 & 0.0614 & 0.2231 \\
& $(0.0030)$ & $(0.0124)$ & $(0.0060)$ & $(0.0145)$ & $(0.0050)$ & $(0.0057)$ \\
$\phi$ & 0.6923 & 0.7845 & -0.0395 & 0.9719 & 0.6532 & 0.9541 \\
& $(0.0208)$ & $(0.0169)$ & $(0.0011)$ & $(0.0200)$ & $(0.0181)$ & $(0.0722)$ \\
$\{s, k\}$ & $\{1,3\}$ & $\{30,0\}$ & $\{1,3\}$ & $\{20,0\}$ & $\{1,4\}$ & $\{20,0\}$ \\
$d$ & 0.4988 & 0.0587 & 0.3817 & 0.0585 & 0.4879 & 0.0400 \\
& $(0.0206)$ & $(0.0013)$ & $(0.0071)$ & $(0.0018)$ & $(0.0086)$ & $(0.0030)$ \\
$d_{1}$ & 0.0145 & & 0.0685 & & 0.0262 & \\
& $(0.0002)$ & & $(0.0022)$ & & $(0.0009)$ & \\
$d_{2}$ & 0.0553 & & -0.0078 & & 0.1262 & \\
& $(0.0014)$ & & $(0.0002)$ & & $(0.0034)$ & \\
$d_{3}$ & -0.1780 & & 0.0288 & & 0.1262 & \\
& $(0.0038)$ & & $(0.0006)$ & & $(0.0027)$ & \\
$d_{4}$ & & & & & -0.4656 & \\
& & & & & & \\
QLR Test & 594.24 & {$[0.0000]$} & 1.9191 & {$[0.3831]$} & 61.438 & {$[0.0000]$} \\
\hline \hline
\end{tabular}

Note: Standard errors are in parentheses. 'QLR Test' reports the statistic for testing the null hypothesis of the RSV-SLM model, which has the asymptotic $\chi^{2}(k)$ distribution. $P$-values are given in brackets.

Table 6: Forecasting Results

\begin{tabular}{c|cccccc}
\hline \hline & \multicolumn{2}{|c}{ S\&P } & \multicolumn{2}{c}{ FTSE } & \multicolumn{2}{c}{ Nikkei } \\
\cline { 2 - 7 } & RMSE & DM & RMSE & DM & RMSE & DM \\
\hline HAR & 6.6671 & - & 3.7408 & - & 3.4874 & - \\
RSV-GGLM & 5.7713 & {$[0.0000]$} & 3.4692 & {$[0.0003]$} & 3.3241 & {$[0.0152]$} \\
RSV-SLM & 5.5445 & {$[0.0000]$} & 3.3676 & {$[0.0000]$} & 3.1617 & {$[0.0008]$} \\
Combined Forecasts & 5.3930 & {$[0.0000]$} & 3.2971 & {$[0.0000]$} & 3.0308 & {$[0.0007]$} \\
\hline \hline
\end{tabular}

Note: The values in the brackets are $P$-values of the Diebold-Mariano test against the forecast via the HAR model. Combined Forecasts are obtained by the weighted average of the forecasts of RSV-GGLM and RSV-SLM models, with weights $(-1,2)$. 
Figure 1: Power Spectrum of Long Memory Processes

(a) Seasonal Long Memory

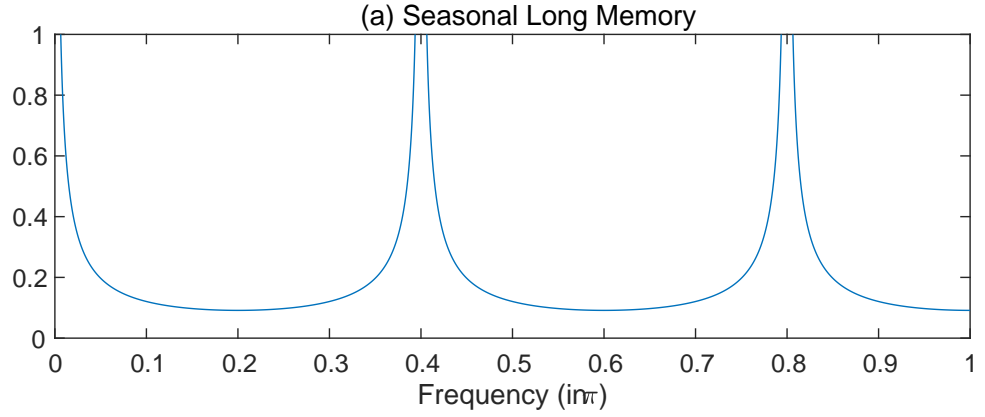

(b) General Long Memory

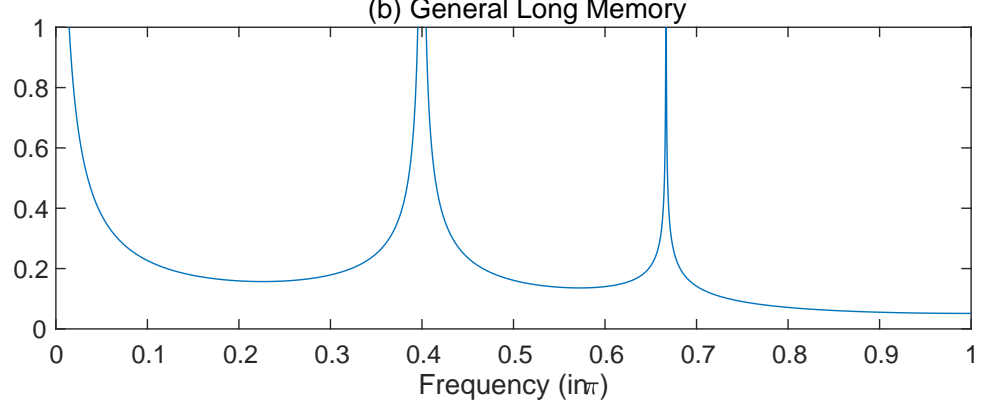


Figure 2: Sample Spectral Density of Log-Volatility

(a) S\&P 500

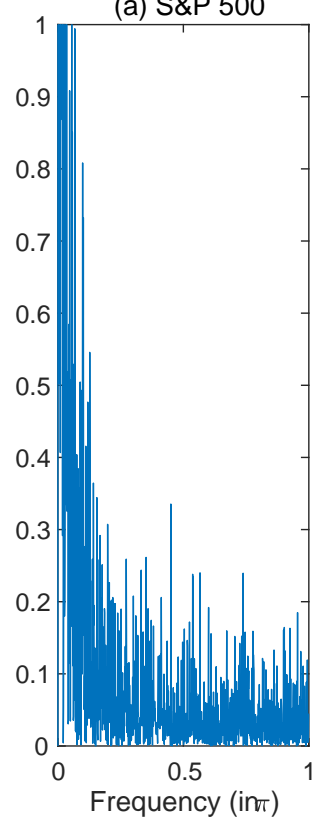

(b) FTSE 100

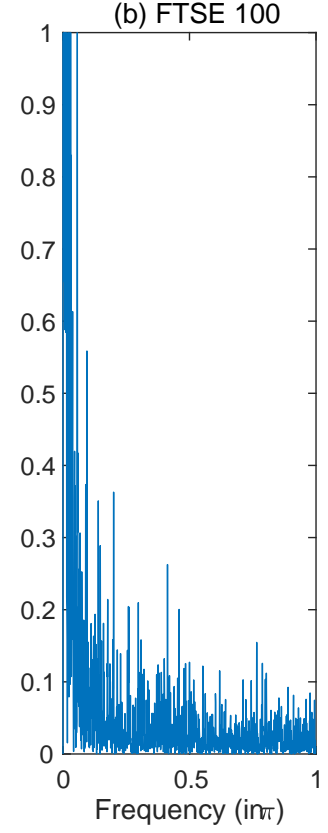

(c) Nikkei 225

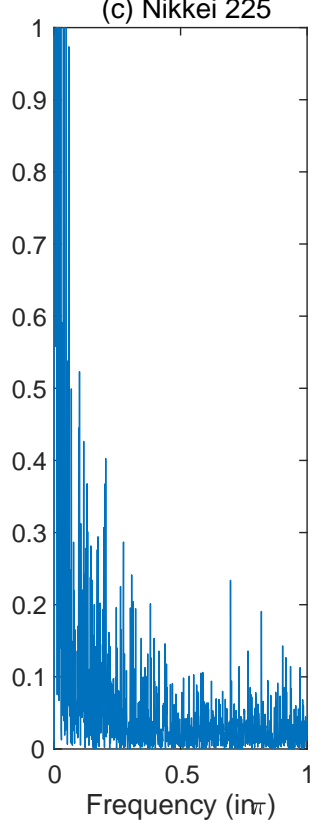

